\title{
Rotationally resolved electronic spectroscopy of water clusters of 7-azaindole
}

\author{
Thi Bao Chau Vu, ${ }^{1}$ Ivo Kalkman, ${ }^{2}$ W. Leo Meerts, ${ }^{2}$ Yuriy N. Svartsov, ${ }^{3}$ Christoph Jacoby, ${ }^{1}$ \\ and Michael Schmitt ${ }^{1, a)}$ \\ ${ }_{1}^{1}$ Institut für Physikalische Chemie I, Heinrich-Heine-Universität, D-40225 Düsseldorf, Germany \\ ${ }^{2}$ Department of Molecular and Laser Physics, Institute for Molecules and Materials, Radboud University \\ Nijmegen, P.O. Box 9010 NL-6500 GL Nijmegen, The Netherlands \\ ${ }^{3}$ Institut für Anorganische Chemie, Johannes Kepler Universität Linz, Altenbergerstraße 69, A-4040 Linz, \\ Austria
}

(Received 1 February 2008; accepted 23 April 2008; published online 6 June 2008)

\begin{abstract}
The rotationally resolved electronic spectra of the electronic origin of the 7-azaindole- $\left(\mathrm{H}_{2} \mathrm{O}\right)_{1}$ and of the 7-azaindole- $\left(\mathrm{H}_{2} \mathrm{O}\right)_{2}$ clusters have been measured in a molecular beam. From the rotational constants the structures in the $S_{0}$ and $S_{1}$ electronic states were determined as cyclic with the pyrrolo $\mathrm{NH}$ and the pyridino $\mathrm{N}$ atoms being bridged by one and two water molecules, respectively. Excited state lifetimes of about $10 \mathrm{~ns}$ for both clusters have been found. In the spectrum of the 7-azaindole- $\left(\mathrm{H}_{2} \mathrm{O}\right)_{2}$ cluster a splitting of the rovibronic band is observed, which can be traced back to a large amplitude motion, involving the out-of-plane hydrogen atoms of the water chain. Both the changes of the rotational constants upon electronic excitation and the orientation of the transition dipole point to a solvent induced state reversal between the $L_{a}$ and the $L_{b}$ states upon microsolvation. ㅇ 2008 American Institute of Physics. [DOI: 10.1063/1.2928637]
\end{abstract}

\section{INTRODUCTION}

In a preceding theoretical study on the lowest electronically excited states of 7-azaindole and the 7-azaindole $\left(\mathrm{H}_{2} \mathrm{O}\right)_{1,2}$ clusters, the geometries, excitation energies, transition dipole orientations, and natures of the excited states have been predicted at the RICC2 and DFT/MRCI level of theory. ${ }^{1}$ In the following some of these theoretical predictions will be tested, using rotationally resolved electronic spectroscopy of the 7-azaindole $\left(\mathrm{H}_{2} \mathrm{O}\right)_{1,2}$ clusters.

Laser spectroscopic investigations of the structures and photophysical properties of azaindole and its hydrogen and van der Waals bound clusters range back to the early 80 s of the last century. Fuke $e t a l^{2}$ measured mass-resolved ionization spectra and fluorescence excitation spectra of 7-azaindole, 7-azaindole $\left(\mathrm{H}_{2} \mathrm{O}\right)_{1,2}$, and (7-azaindole $)_{2}$. From the large spectral redshift $\left(1285 \mathrm{~cm}^{-1}\right)$ they proposed a considerable distortion of the geometry upon electronic excitation of the water clusters.

Kim and Bernstein ${ }^{3}$ presented mass-resolved excitation spectra of 7-azaindole and its clusters with $\mathrm{Ar}, \mathrm{CH}_{4}, \mathrm{NH}_{3}$, $\mathrm{H}_{2} \mathrm{O}, \mathrm{D}_{2} \mathrm{O}, \mathrm{CH}_{3} \mathrm{OH}$, and $\mathrm{C}_{2} \mathrm{H}_{5} \mathrm{OH}$. For the azaindole-water cluster they deduced a structure in which the water moiety is bound to the $\pi$-system instead of a hydrogen bonded structure.

Nakajima et al. ${ }^{4}$ investigated the geometric structures of 7-azaindole $\left(\mathrm{H}_{2} \mathrm{O}\right)_{1-3}$ and of the 7-azaindole dimer using laser-induced fluorescence spectroscopy with high resolution $\left(\sim 0.01 \mathrm{~cm}^{-1}\right.$, equivalent to $\left.300 \mathrm{MHz}\right)$. They deduced a planar ring structure of 7 -azaindole $\left(\mathrm{H}_{2} \mathrm{O}\right)_{1}$ from the rotational analysis of the spectrum and from $a b$ initio calculations. For

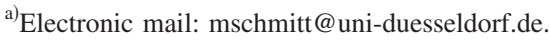

the $n=2$ and 3 clusters, also cyclic hydrogen bonded ring structures with the water oxygen atoms in the aromatic plane were suggested.

In the work of Fomer et al. pump-probe experiments have been performed on the $n=2,3$, and 4 water clusters of azaindole, which yield for the doubly and triply hydrated clusters biexponential decays with a short component (a few hundred femtoseconds) and a long component (6 ps). They attribute this behavior to a stepwise proton transfer from the $1 H$ to the $7 H$ position in the $n=2$ and 3 cluster, while a single exponential decay for the $n=4$ cluster was believed to be the signature of a concerted proton transfer. ${ }^{5}$

Yokoyama et al. performed IR-UV double resonance spectroscopy on 7-azaindole-water clusters together with $a b$ initio molecular orbital calculations. ${ }^{6}$ From their results hydrogen bonded single ring structures for the 7 -azaindole $\left(\mathrm{H}_{2} \mathrm{O}\right)_{n}$ cluster with $n=1-3$ was deduced in the electronic ground state.

The structural changes of the 7-azaindole $\left(\mathrm{H}_{2} \mathrm{O}\right)_{1}$ cluster upon electronic excitation were determined from a FranckCondon analysis of the emission spectra obtained via pumping of several vibronic bands. ${ }^{7}$ The distances of both the $\mathrm{O} \cdots \mathrm{H}$ and the $\mathrm{H} \cdots \mathrm{N}$ hydrogen bond are found to decrease upon electronic excitation, accompanied by a distortion of the monomer geometry.

Dispersed fluorescence spectra of 7-azaindole $\left(\mathrm{H}_{2} \mathrm{O}\right)_{1-3}$ clusters were presented by Hara et al. ${ }^{8}$ No visible emission from the proton transferred tautomers could be observed in their experiment, excluding the excited state proton transfer (ESPT) taking place in the gas phase on a time scale comparable to the fluorescence lifetime.

The geometrical changes of the 7-azaindole monomer itself were investigated using rotationally resolved electronic 


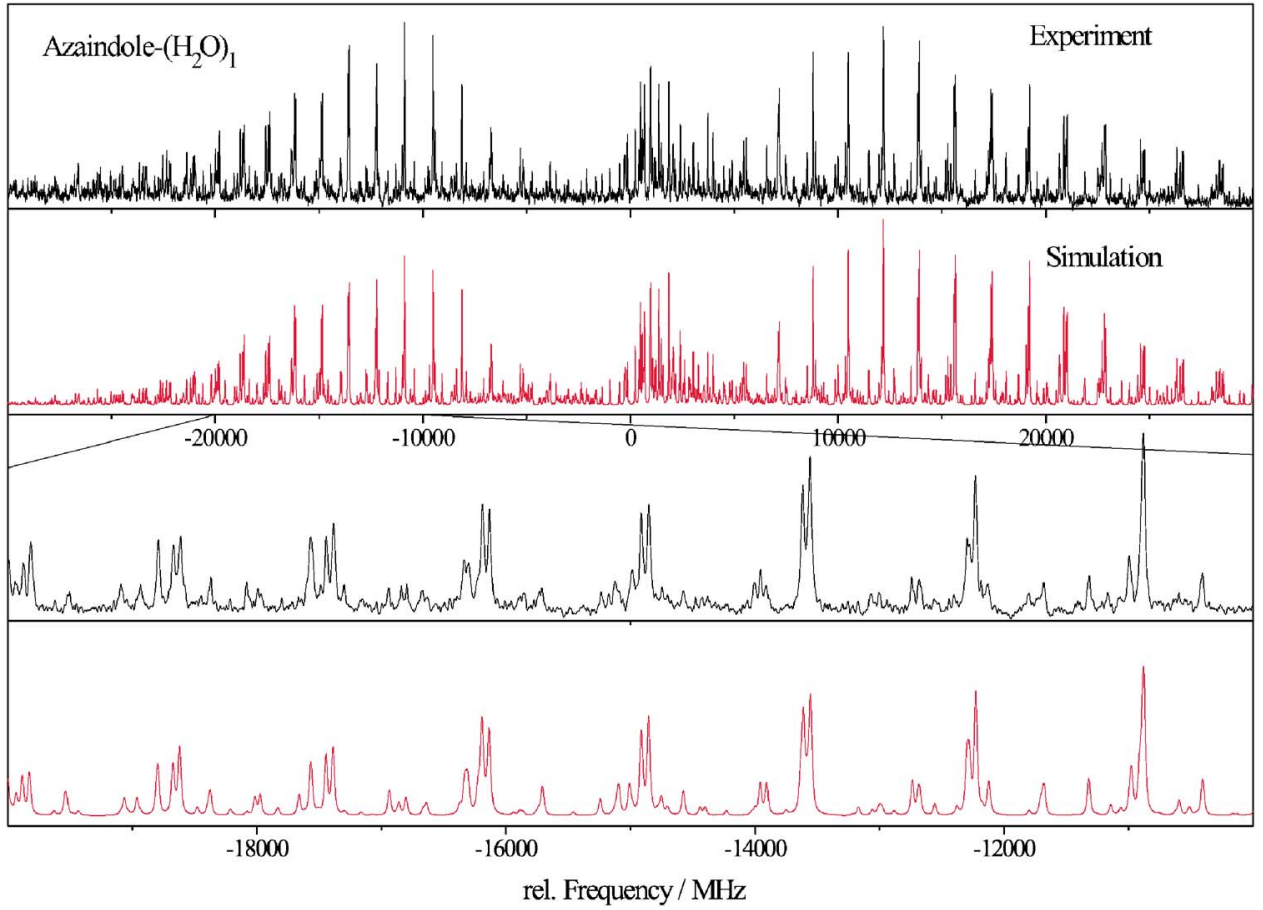

FIG. 1. (Color online) Rotationally resolved spectrum of the electronic origin of the 7-azaindole- $\left(\mathrm{H}_{2} \mathrm{O}\right)_{1}$ water cluster and simulation of the spectrum using the molecular parameters from the best GA fit, given in Table I. The lower two traces show an expanded view in the range of -20000 to $-10000 \mathrm{MHz}$ relative to the electronic origin.

spectroscopy of four different isotopomers of 7-azaindole 9,10 as well as a Franck-Condon analysis. ${ }^{11}$ In Ref. 9 the nature of the electronically excited state has been shown to be predominantly of $L_{b}$ character. Recently, the excited state dipole moment of 7-azaindole has been determined by Kang et al. from the Stark effect in a rotationally resolved LIF spectrum. $^{12}$

\section{EXPERIMENTAL METHODS}

The experimental setup for the rotationally resolved laser-induced fluorescence is described in detail elsewhere. ${ }^{13}$ Briefly, it consists of a ring dye laser (Coherent 899-21) operated with Kiton Red, pumped with $7 \mathrm{~W}$ of the frequency doubled output of a diode pumped Yb:YAG disc laser (TCFKA ELS). About 600-700 $\mathrm{mW}$ of the fundamental dye laser output is coupled into an external folded ring cavity (Spectra Physics) for second harmonic generation.

The molecular beam is formed by expanding azaindole heated to $100{ }^{\circ} \mathrm{C}$ and seeded in $300-700$ mbars of argon through a $100 \mu \mathrm{m}$ hole into the vacuum. The molecular beam machine consists of three differentially pumped vacuum chambers that are connected by two skimmers ( 1 and $3 \mathrm{~mm}$, respectively) in order to reduce the Doppler width to $25 \mathrm{MHz}$. The molecular beam is crossed at right angles in the third chamber with the laser beam $360 \mathrm{~mm}$ downstream of the nozzle. The resulting fluorescence is collected perpendicular to the plane defined by laser and molecular beam by an imaging optics setup consisting of a concave mirror and two plano-convex lenses. The fluorescence is detected by a UV enhanced photomultiplier tube whose output is recorded by a PC based photon counter IO card. The relative frequency is determined with a quasiconfocal Fabry-Perot interferometer. The absolute frequency was determined by recording the iodine absorption spectrum and comparison the transitions to the tabulated lines. ${ }^{14}$

\section{EXPERIMENTAL RESULTS}

\section{A. 7-azaindole $\left(\mathrm{H}_{2} \mathrm{O}\right)_{1}$}

Figure 1 shows the rotationally resolved spectrum of the electronic origin of 7-azaindole- $\left(\mathrm{H}_{2} \mathrm{O}\right)_{1}$. The spectrum spans about $60 \mathrm{GHz}$ and displays a well resolved rovibronic structure with predominantly $a b$-hybrid type.

The molecular parameters obtained from a genetic algorithm (GA) based automated fit of the spectrum are given in Table I, and are compared to the results of various ab initio calculations for both electronic states. For details about the fitting procedure using the GA technique see Refs. 15-17. Although, as in the case of the azaindole monomer, ${ }^{9}$ a simple rigid rotor Hamiltonian without axis reorientation was employed, all intensities and line positions are well reproduced (cf. Fig. 1). The slightly negative inertial defect $\Delta I$ and the negligible change of $\Delta I$ upon electronic excitation $(\Delta \Delta I)$ point to nearly planar structures with at most one hydrogen atom pointing out of the molecular plane in both electronic states. The transition dipole moment, determined from the relative intensities of $a$ and $b$ lines in the spectrum, makes an angle of $\pm 27^{\circ}$ with the inertial $a$ axis, slightly different from the value, which has been obtained from a fit to the rovibronic contour $\left( \pm 16^{\circ}\right)$ taken at much lower resolution $(300 \mathrm{MHz}){ }^{7}$ The polar angle $\phi$, which introduces $c$-type character in the vibronic band was determined to be $\pm 86^{\circ}$, meaning less than $1 \% c$ type. Thus, the transition dipole moment is located in the plane of the chromophore. For the discussion of the sign of the TDM angle $\theta$, cf. Sec. IV B. The line shape was fitted to a Voigt profile with a Doppler contribution of $25 \mathrm{MHz}$, resulting in a Lorentz contribution of $15 \pm 4 \mathrm{MHz}$.

The agreement between the experimental and computed rotational constants in both electronic states is very good for the time dependent density functional theory and the coupled cluster RICC2/TZVP ab initio calculations from Ref. 1 
TABLE I. Comparison of the molecular parameters from the fit to the rotationally resolved electronic spectrum of 7-azaindole $\left(\mathrm{H}_{2} \mathrm{O}\right)_{1}$ shown in Fig. 1 to the results of $a b$ initio calculations. (TD)DFT and RICC2 were performed with the TZVP basis. For details of the DFT/MRCI calculations, cf. part I of the present study (Ref. $1)$.

\begin{tabular}{|c|c|c|c|c|c|}
\hline & Expt. & (TD)B3-LYP & $\operatorname{RICC}^{\mathrm{a}}$ & DFT/MRCI ${ }^{\mathrm{a}}$ & $\operatorname{CAS}(10,9)^{\mathrm{b}}$ \\
\hline$A^{\prime \prime}(\mathrm{MHz})$ & 1761.75 & 1775 & 1784 & $1784^{\mathrm{c}}$ & 1780 \\
\hline$B^{\prime \prime}(\mathrm{MHz})$ & 1342.80 & 1349 & 1353 & $1353^{\mathrm{c}}$ & 1288 \\
\hline$C^{\prime \prime}(\mathrm{MHz})$ & 762.89 & 768 & 771 & $771^{\mathrm{c}}$ & 749 \\
\hline$\Delta I\left(\mathrm{amu} \AA^{2}\right)$ & -0.77 & -1.31 & -1.04 & -1.04 & -1.56 \\
\hline$\theta\left({ }^{\circ}\right)$ & \pm 27 & -17 & -3 & +19 & -89 \\
\hline$\phi\left({ }^{\circ}\right)$ & \pm 86 & +90 & +90 & +89 & +90 \\
\hline$\mu_{a}^{2}$ & 0.777 & 0.914 & 0.995 & 0.896 & 0.000 \\
\hline$\mu_{b}^{2}$ & 0.217 & 0.085 & 0.005 & 0.104 & 1.000 \\
\hline$\mu_{c}^{2}$ & 0.006 & 0.000 & 0.000 & 0.000 & 0.000 \\
\hline$\nu_{0}\left(\mathrm{~cm}^{-1}\right)$ & 33340.43 & 32360 & 33288 & 33214 & 37320 \\
\hline$\Delta A(\mathrm{MHz})$ & 35.97 & 25 & 35 & 35 & $-13^{\mathrm{c}}$ \\
\hline$\Delta B(\mathrm{MHz})$ & 15.34 & 15 & 14 & 14 & $-22^{\mathrm{c}}$ \\
\hline$\Delta C(\mathrm{MHz})$ & 11.80 & 9 & 11 & 11 & $-10^{\mathrm{c}}$ \\
\hline$\Delta \Delta I\left(\mathrm{amu} \AA^{2}\right)$ & -0.09 & 0.45 & -0.15 & -0.15 & 0.23 \\
\hline
\end{tabular}

${ }^{\mathrm{a} C a l c u l a t i o n s ~ d e s c r i b e d ~ i n ~ R e f . ~} 1$.

${ }^{\mathrm{b}}$ Calculations described in Ref. 7.

${ }^{\mathrm{c}}$ Structure used for the DFT/MRCI calculations is the RICC2/TZVP relaxed structure.

which are also given in Table I for comparison. The complete active space CAS $(10,9) / c c-p V D Z$ calculations ${ }^{7}$ agree well in the electronic ground state, but even the signs of the changes of the rotational constants upon electronic excitation are predicted wrongly for all rotational constants at this level of theory. The rotational constants reported for DFT/MRCI calculations are the ones of the relaxed RICC2 geometry, at which DFT/MRCI has been performed. ${ }^{1}$

The transition properties such as excitation energies and transition dipole moments are quite satisfactorily reproduced at RICC2/TZVP and even better at DFT/MRCI level, while the CASSCF calculations predict the band type completely wrong, and also the adiabatic excitation energy, which is off by nearly $4000 \mathrm{~cm}^{-1}$.

\section{B. 7-azaindole $\left(\mathrm{H}_{2} \mathrm{O}\right)_{2}$}

Due to the large oscillator strength of transitions to the lowest electronically excited state of 7-azaindole, and the large stability of the hydrogen bonded water clusters, also higher water clusters can be observed with rotationally resolved electronic spectroscopy. Figure 2 shows the spectrum of the electronic origin of the 7-azaindole $\left(\mathrm{H}_{2} \mathrm{O}\right)_{2}$ cluster. In contrast to the case of the 1:1 cluster, the spectrum of the $1: 2$ cluster consists of two closely spaced components, with a splitting of $29469 \mathrm{MHz}$, which can easily be determined in an autocorrelation of the spectrum. The spectrum has been fit using a rigid rotor Hamiltonian without axis reorientation, for each of the components using the GA technique, as for the $1: 1$ water cluster.

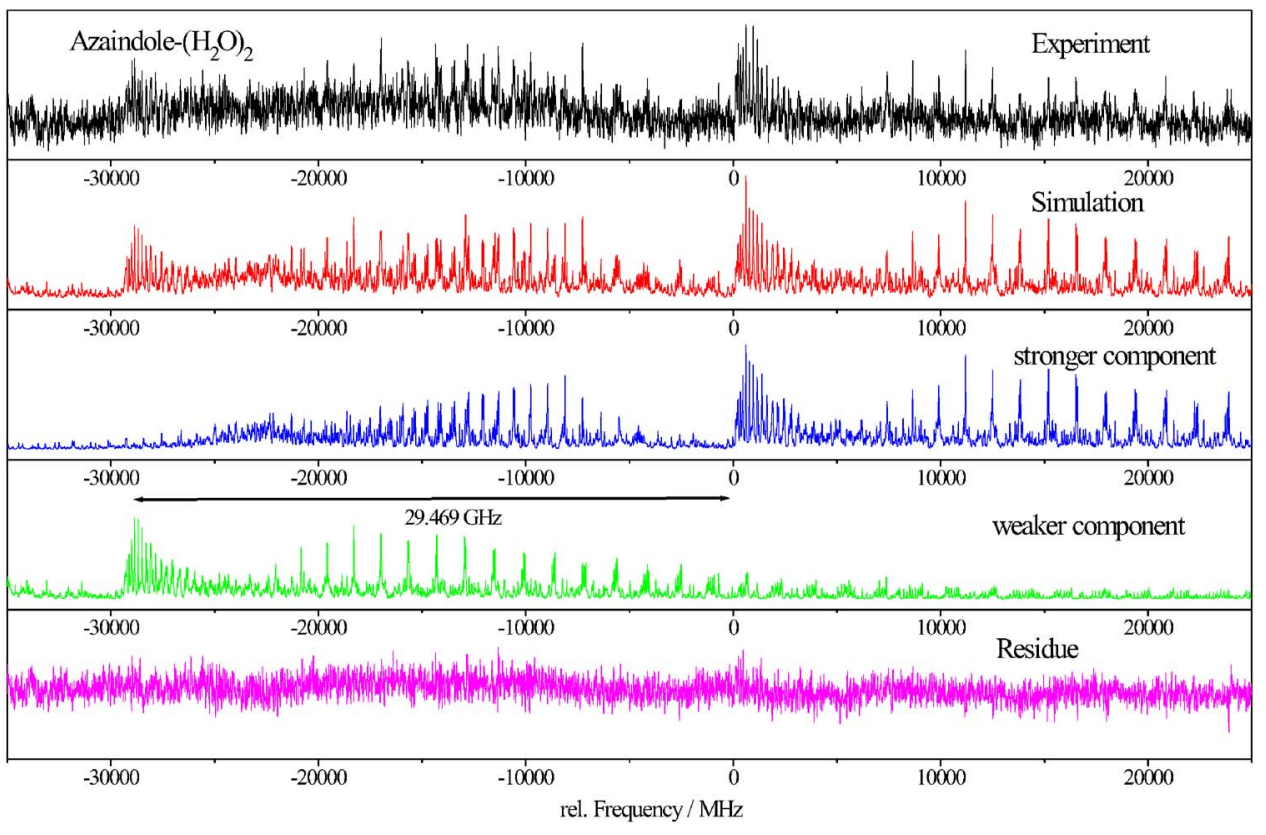

FIG. 2. (Color online) Rotationally resolved spectrum of the electronic origin of 7-azaindole $\left(\mathrm{H}_{2} \mathrm{O}\right)_{2}$ and simulation of the spectrum using the molecular parameters from the best GA fit, given in Table II. The last trace shows the residue of the sum of the individual fits of the subcomponents and the experimental spectrum. Frequencies in megahertz are given relative to the origin of the stronger component. 
TABLE II. Comparison of the molecular parameters from the fit with the rotationally resolved electronic spectrum of 7-azaindole $\left(\mathrm{H}_{2} \mathrm{O}\right)_{2}$ shown in Fig. 2 to the results of ab initio calculations. The (TD)DFT and RICC2 calculations are performed with the TZVP basis. For details of the calculations, cf. part I of the present study (Ref. 1).

\begin{tabular}{lccccc}
\hline \hline & Expt. band 1 & Expt. band 2 & (TD)B3-LYP & RICC2 & DFT/MRCI \\
\hline$A^{\prime \prime}(\mathrm{MHz})$ & $1413.81(25)$ & $1415.46(52)$ & 1424 & 1425 & 1425 \\
$B^{\prime \prime}(\mathrm{MHz})$ & $827.61(9)$ & $828.00(18)$ & 856 & 842 & 842 \\
$C^{\prime \prime}(\mathrm{MHz})$ & $524.02(5)$ & $524.15(8)$ & 537 & 533 & 533 \\
$\Delta I\left(\mathrm{amu} \AA^{2}\right)$ & -3.7 & -3.2 & -5.0 & -6.9 & -6.9 \\
$\theta\left({ }^{\circ}\right)$ & \pm 34 & \pm 35 & -22 & +16 & +33.7 \\
$\phi\left(^{\circ}\right)$ & \pm 74 & \pm 66 & +90 & +90 & +87.1 \\
$\mu_{a}^{2}$ & 0.6336 & 0.5594 & 0.8536 & 0.9200 & 0.690 \\
$\mu_{b}^{2}$ & 0.2956 & 0.2805 & 0.1463 & 0.0799 & 0.307 \\
$\mu_{c}^{2}$ & 0.0708 & 0.1600 & 0.0001 & 0.0001 & 0.003 \\
$\nu_{0}\left(\mathrm{~cm}^{-1}\right)$ & 32632.10 & $\ldots$ & 32850 & 29358 & 32751 \\
$\Delta \nu_{0}(\mathrm{MHz})$ & $\ldots$ & 29469 & $\ldots$ & $\ldots$ & $\ldots$ \\
$\Delta A(\mathrm{MHz})$ & $9.35(3)$ & $9.47(2)$ & +30 & +5 & +5 \\
$\Delta B(\mathrm{MHz})$ & $27.54(2)$ & $27.38(5)$ & +31 & +28 & +28 \\
$\Delta C(\mathrm{MHz})$ & $12.59(2)$ & $12.48(1)$ & +16 & +16 & +16 \\
$\Delta \Delta I\left(\mathrm{amu} \AA^{2}\right)$ & -0.6 & -0.5 & -0.3 & -0.7 & -0.7 \\
\hline \hline
\end{tabular}

The rotational constants for both electronic states are given in Table II and are compared to the results of ab initio calculations. The agreement with the results of the time dependent DFT and the RICC2 calculations is as satisfying as for the $n=1$ cluster. The considerably larger inertial defect in both electronic states points to a slightly out-of-plane geometry for at least one of the heavy atoms. Comparison to the calculated structures shows that the oxygen atoms of both water moieties in the hydrogen bond chain are slightly out of plane-one being below, the other above the aromatic plane.

As can be already inferred from the strong and unusually sharp correlation side peak at a shift of $29469 \mathrm{MHz}$ relative to the unshifted autocorrelation, the rotational constants of the two subbands are very similar, cf. Table II. The nature of the large amplitude motion, responsible for the splitting in the spectrum will be investigated in detail in Sec. IV D.

The characteristics of the electronic excitation (adiabatic excitation energy and orientation of the transition dipole moment orientation are again well reproduced at the DFT/ MRCI level of theory. The angle $\theta$ between the TDM and the $a$ axis is determined to be $\pm 34^{\circ}$ from the intensities in the experimental spectrum and to +33.7 from the DFT/MRCI calculations.

As for the 7-azaindole $\left(\mathrm{H}_{2} \mathrm{O}\right)_{1}$ cluster, the line shape was fitted to a Voigt profile with a Doppler contribution of $25 \mathrm{MHz}$, resulting in a Lorentz contribution of $15 \pm 6 \mathrm{MHz}$.

\section{Determination of the cluster structures}

The partial intermolecular structures of the $n=1,2$ clusters have been determined from the rotational constants given in Tables I and II using the program PKRFIT. ${ }^{18,19}$ The structures of the 7-azaindole monomer in both electronic states was taken from a combined structural fit to rotational constants of four different isotopomers and to the FranckCondon intensities of 107 emission bands. ${ }^{11}$ The $\mathrm{OH}$ distance in the water moiety was set to $96 \mathrm{pm}$, and the water $\mathrm{HOH}$ angle to $104^{\circ}$. Only three geometry parameters could be determined, because for each cluster the rotational constants of only one isotopomer have been measured. For the $n=1$ cluster, the $\mathrm{H}_{1} \mathrm{O}_{8}$ bond length, the $\mathrm{N}_{1} \mathrm{H}_{1} \mathrm{O}_{8}$ angle, and the $\mathrm{N}_{1} \mathrm{H}_{1} \mathrm{O}_{8} \mathrm{H}_{8 b}$ dihedral angle have been chosen. The definitions of the distances, angles, and dihedral angles are given in Fig. 3. The second hydrogen bond length $\mathrm{N}_{7} \mathrm{H}_{8 a}$ was not included in the fit, but is obtained implicitly from the definition of the $Z$ matrix used in the fit. A distinct shortening of both hydrogen bonds upon electronic excitation is found.

Table III presents the results of the fit. In addition to the three fitted parameters, the other three values which determine the intermolecular geometry are given for completeness.

For the $n=2$ cluster the restriction of the geometry model due to the small number of inertial parameters is even more unsatisfying. As for the binary cluster, the geometry of the monomer for both electronic states was taken from the Franck-Condon analysis presented in Ref. 11. Two hydrogen bond distances and the angle, which describes the deviation of the $\mathrm{N}-\mathrm{H}_{1} \cdots \mathrm{O}_{8}$ hydrogen bond from linearity were fitted.
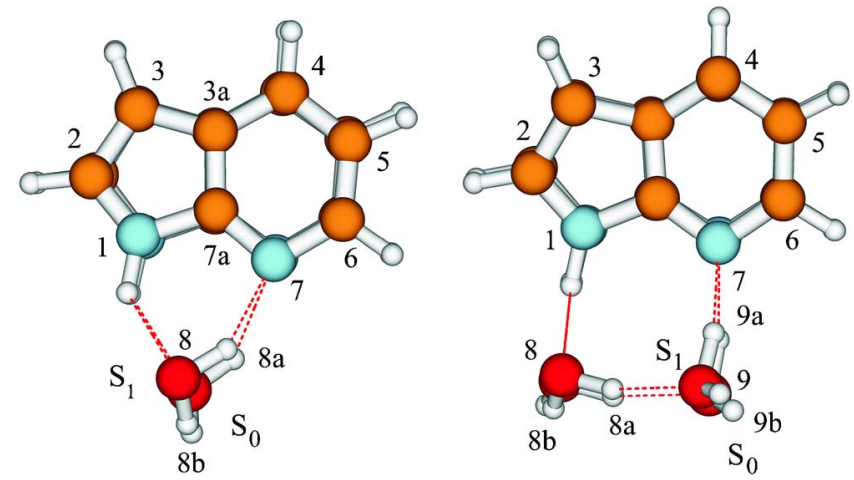

FIG. 3. (Color online) Structures of 7-azaindole $\left(\mathrm{H}_{2} \mathrm{O}\right)_{1}$ and 7-azaindole $\left(\mathrm{H}_{2} \mathrm{O}\right)_{2}$ in the ground and first excited singlet states from the fit to the rotational constants. The structures for both states are overlayed, with a maximum of congruence for the azaindole moiety. In each case, the upper structure shows the excited state. 
TABLE III. $S_{0}$ and $S_{1}$ state geometry parameters $\left(r_{0}\right)$ of the 7-azaindole $\left(\mathrm{H}_{2} \mathrm{O}\right)_{1,2}$ clusters, from the fit to the experimental rotational constants. All bond lengths are given in picometers, angles and dihedral angles are given in degrees.

\begin{tabular}{|c|c|c|c|c|c|}
\hline \multirow[b]{2}{*}{$r_{0}$} & \multicolumn{2}{|c|}{ 7- $\mathrm{AI}\left(\mathrm{H}_{2} \mathrm{O}\right)_{1}$} & \multirow[b]{2}{*}{$r_{0}$} & \multicolumn{2}{|c|}{ 7-AI $\left(\mathrm{H}_{2} \mathrm{O}\right)_{2}$} \\
\hline & $S_{0}$ & $S_{1}$ & & $S_{0}$ & $S_{1}$ \\
\hline $\mathrm{H}_{1} \mathrm{O}_{8}{ }^{\mathrm{a}}$ & 215.3 & 195 & $\mathrm{H}_{1} \mathrm{O}_{8}{ }^{\mathrm{b}}$ & 175.2 & 169.3 \\
\hline $\mathrm{N}_{1} \mathrm{H}_{1} \mathrm{O}_{8}{ }^{\mathrm{a}}$ & 131 & 127 & $\mathrm{H}_{8 a} \mathrm{O}_{9}{ }^{\mathrm{a}}$ & 170.2 & 167.9 \\
\hline $\mathrm{N}_{1} \mathrm{H}_{1} \mathrm{O}_{8} \mathrm{H}_{8 b}{ }^{\mathrm{a}}$ & 139 & 95 & $\mathrm{O}_{8} \mathrm{H}_{1} \mathrm{~N}_{1}^{\mathrm{a}}$ & 167 & 162 \\
\hline $\mathrm{N}_{7} \mathrm{H}_{8 a}$ & 228.1 & 211.7 & $\mathrm{O}_{9} \mathrm{H}_{8 a} \mathrm{O}_{8}^{\mathrm{b}}$ & 167 & 162 \\
\hline $\mathrm{N}_{7} \mathrm{H}_{8 a} \mathrm{O}_{8}$ & 116 & 124 & $\mathrm{H}_{9 a} \mathrm{~N}_{7}$ & 214.1 & 181.8 \\
\hline $\mathrm{H}_{1} \mathrm{O}_{8} \mathrm{H}_{8 b}$ & 110 & 110 & $\mathrm{H}_{9 a} \mathrm{~N}_{7} \mathrm{O}_{9}$ & 156 & 166 \\
\hline
\end{tabular}

${ }^{\mathrm{a}}$ Used in the fit.

${ }^{b}$ The angle $\mathrm{O}_{9} \mathrm{H}_{8 a} \mathrm{O}_{8}$ was kept fix at the fitted value of $\mathrm{O}_{8} \mathrm{H}_{1} \mathrm{~N}_{1}$.

Again, the last hydrogen bond length $\mathrm{H}_{9 a} \mathrm{~N}_{7}$ results automatically from the definition of the internal coordinates. As in the case of the 1:1 cluster, all hydrogen bond lengths decrease upon electronic excitation. Spectra of a much larger number of isotopomers will be necessary to completely unravel the structure of the $n=2$ cluster in both electronic states in the future.

\section{DISCUSSION}

\section{A. The structures of the clusters in ground and excited states}

The comparison to calculated rotational constants shows, that both for the ground state and the excited state the $1 H$ tautomer is experimentally observed, although the $7 H$ tautomer is found to be considerably more stable in the $S_{1}$ state. Comparison to the results of the RICC2/TZVP calculations in Ref. 1 shows a large change of the geometry of the azaindole monomer moiety upon water complexation in the electronically excited state, while the ground state geometry of 7-azaindole in the monomer and the water cluster is nearly the same. The reason for this strange behavior in the excited state is a solvent induced state reversal between the $L_{b}$ and $L_{a}$ states, the former being the $S_{1}$ state in the monomer, the latter in the 1- and 2-water clusters. Of special interest for future experiments will be the determination of selected geometry parameters of the monomer in the cluster, using isotopically substituted species and Kraitchmans equations. These present a direct access to the question of the electronic nature of the excited states.
Both hydrogen bond lengths in the 7-azaindole $\left(\mathrm{H}_{2} \mathrm{O}\right)_{1}$ cluster $\left(\mathrm{H}_{1} \mathrm{O}_{8}\right.$ and $\left.\mathrm{N}_{7} \mathrm{H}_{8 a}\right)$ are considerably shortened upon electronic excitation which can be explained by the increased excited state acidity of the pyrrole moiety and the increased basicity of the pyridine moiety in the first electronically excited $\pi-\pi^{*}$ state. $^{20}$ Figure 3 shows the simultaneous decrease of both hydrogen bonds in the excited state.

For the 7-azaindole $\left(\mathrm{H}_{2} \mathrm{O}\right)_{2}$ cluster, the $\mathrm{H}_{1} \mathrm{O}_{8}$ bond length also decreases upon electronic excitation. The hydrogen bond length between the two water moieties $\mathrm{H}_{8 a} \mathrm{O}_{9}$ decreases slightly, due to polarization effects on both water moieties, mediated by the basicity increase of the pyridino $\mathrm{N}$ atom and the increase in acidity of the pyrrolo $\mathrm{N}(\mathrm{H})$ site. $^{21}$

\section{B. Orientation of the transition dipole}

The orientation of the transition dipole moment in the 7-azaindole monomer was experimentally determined to be $-14^{\circ}$ (Ref. 10) or $-20^{\circ}$ (Ref. 9) with respect to the $a$ axis. The minus sign refers to a orientation, in which the TDM points in direction of the pyrrolic NH group [orientation I in Fig. 4(a)]. The DFT/MRCI calculations, reported in part I (Ref. 1) of the present investigation, however, predict a TDM orientation of $+20^{\circ}$ [orientation II in Fig. 4(a)].

The orientation of the TDM in the water cluster is determined in the present work to be $\pm 27^{\circ}$ with respect to the $a$ axis of the cluster. The RICC2/TZVP calculations, reported in part I (Ref. 1) which reproduce the experimental excited state rotational constants better than $1 \%$, show that the angle of the inertial $a$-axis in the cluster with the $\mathrm{C}_{3 a} \mathrm{C}_{7 a}$ bond (cf. (a)

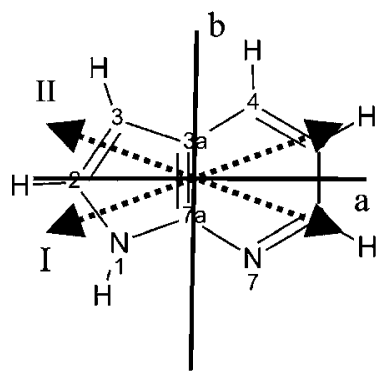

(b)

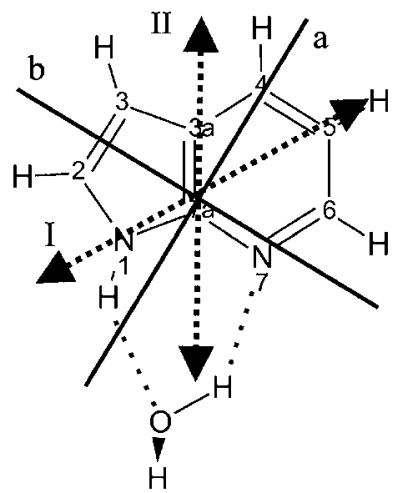

(c)

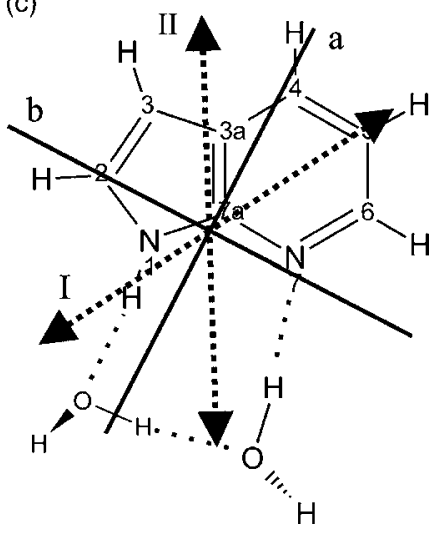

FIG. 4. Inertial axis systems and TDM orientations of 7-azaindole, 7-azaindole $\left(\mathrm{H}_{2} \mathrm{O}\right)_{1}$, and 7-azaindole $\left(\mathrm{H}_{2} \mathrm{O}\right)_{2}$. 
Fig. 4) is $30^{\circ}$. In the monomer the angle between the $a$ axis and the $\mathrm{C}_{3 a} \mathrm{C}_{7 a}$ bond is found to be $90^{\circ}$. Therefore, the inertial axis system in the frame of the azaindole monomer is rotated counterclockwise by $60^{\circ}$ upon cluster formation, as shown in Fig. 4. The inertial axes of the monomer and the water clusters are shown with the azaindole monomer moieties oriented equally in Fig. 4.

If the positive sign of the TDM orientation in the $n=1$ cluster applies, as predicted by the DFT/MRCI calculations, the TDM would make an angle with the $a$ axis of the monomer of $-33^{\circ}$ [orientation I in Fig. 4(b)]. If on the other hand the negative sign would apply, then the angle of the TDM with the $a$ axis in the monomer geometry would be $87^{\circ}$ (orientation II). This orientation is completely different from both orientations I and II in the 7-azaindole monomer.

For the $n=2$ cluster the rotation of the inertial axes upon water complexation is calculated from the geometries of the RICC2 calculations to be $-70^{\circ}$ [cf. Fig. 4(c)]. The experimentally determined angle of the TDM with the $a$ axis in the inertial system of the cluster is $\pm 34^{\circ}$, while DFT/MRCI predict in very good numerical agreement a value of $+35^{\circ}$. In the inertial system of the monomer, this would correspond to angles of $-35^{\circ}$ for the $+\operatorname{sign}$ (close to orientation $I$ in the monomer) or to $105^{\circ}$ for the - sign (different from both orientations I and II). Again, the positive direction gives an orientation, similar to orientation I in the monomer.

Comparison of the experimental rotational constants of the azaindole monomer and the water clusters to the results of the RICC2 calculations for the lowest excited states showed that the excited state of the clusters has a completely different geometry from that of the monomer, while the ground state geometry is similar. ${ }^{1}$ This points to a change of the ordering of the lowest two excited states upon cluster formation.

Summarizing these findings, it appears as if the TDM angle in the monomer should have a positive sign, as predicted by the DFT/MRCI calculations, but in contradiction to previous experiments. Since these experiments were performed independently in two different groups, the reason for this discrepancy is not easily allegeable. Maybe the assumption made in Refs. 9 and 10 in the interpretation that deuteration leaves the molecule electronically unchanged and rotates only the inertial axes is not correct. If one looks into the theoretical results for the other tautomer of 7-azaindole (the $\mathrm{H}$ being at the 7-position instead of the 1-position), the direction of the TDM is reversed between $L_{a}$ and $L_{b}$ compared to the "normal" $1 H$ tautomer. If we now assume tunneling between the two positions $\left(\mathrm{N}_{1}\right.$ and $\left.\mathrm{N}_{7}\right)$ to occur, the resulting TDM orientation might deviate from that of the pure $1 H$-7-azaindole. Since the barrier is high, most of the contribution to the TDM comes from the "normal" tautomer, but a small fraction is induced by the other tautomer. In the case of the deuterated isotopomer, tunneling is quenched, and the orientation is governed exclusively by the one of the "normal" tautomer. This means that the reason for the change of the angle might be different from what was assumed in both analyses (a pure effect of deuteration on the inertial axes).

\section{Excited state lifetimes}

The Voigt profiles of single rovibronic lines in the 7-azaindole $\left(\mathrm{H}_{2} \mathrm{O}\right)_{1}$ and 7-azaindole $\left(\mathrm{H}_{2} \mathrm{O}\right)_{2}$ spectra exhibit both Lorentz components of $15 \pm 5 \mathrm{MHz}$. This broadening corresponds to an excited state life time of about $10 \mathrm{~ns}$. This value is considerably larger than the excited state life time in the 7-azaindole monomer of 2.5 ns. ${ }^{9}$ Huang et al. reported lifetimes of $8.1 \mathrm{~ns}$ for 7-azaindole $\left(\mathrm{H}_{2} \mathrm{O}\right)_{1}$ and of $6.6 \mathrm{~ns}$ for 7-azaindole $\left(\mathrm{H}_{2} \mathrm{O}\right)_{2}$ using time-correlated single-photon counting. ${ }^{22}$ Folmer et al. found a biexponential decay with time constants of 360 and 6 ps in the excited state of 7-azaindole $\left(\mathrm{H}_{2} \mathrm{O}\right)_{2}$ using pump-probe spectroscopy. ${ }^{5}$ They suggested that the existence of two different time constants points to a stepwise proton transfer reaction in the excited state. Later, Hara et al. showed that, in the fluorescence emission spectra of the 7-azaindole $\left(\mathrm{H}_{2} \mathrm{O}\right)_{1-3}$ clusters in a molecular beam, no visible fluorescence could be detected. ${ }^{8}$ From that, they concluded that ESPT does not occur on the time scale of direct (resonant) fluorescence emission in the gasphase. Our present investigations sustain this conclusion. The much shorter transients, shown in Fig. 2 of Ref. 5, are recorded only up to $7 \mathrm{ps}$. Thus, it is possible that a much longer time component is contained in their transient. By-eye extrapolation of the data in Fig. 2 of Ref. 5 points to a remaining intensity offset, which might be a reminiscence of the slow component, observed in our cw experiment. Alternatively, the transients, which were observed on the different mass channels of the clusters in Ref. 5 might be contaminated by fragmentation from higher cluster sizes, thus yielding an averaged lifetime of many cluster sizes, including very large ones.

\section{Internal motion in the 7-azaindole $\left(\mathrm{H}_{2} \mathrm{O}\right)_{2}$ cluster}

The splitting, which is observed in the spectrum of the 7-azaindole $\left(\mathrm{H}_{2} \mathrm{O}\right)_{2}$ cluster, might be caused by the existence of two different conformers of the cluster, with a small spectral shift due to different zero-point-energy differences. Regarding the fact that the splitting is very small, the chromophore of the two conformers and its close vicinity must be very similar. One possibility for two different conformers in the 7-azaindole $\left(\mathrm{H}_{2} \mathrm{O}\right)_{2}$ cluster is shown in Fig. 5. Here, we plotted the potential energy for the tunneling motion, which interchanges the up and down hydrogen atoms in the water chain, projected to a one-dimensional reduced coordinate. The line of view is from the central hydrogen bond $\left(\mathrm{H}_{8 a} \cdots \mathrm{O}_{9}\right)$ to the azaindole moiety, oriented as shown in Fig. 3. The global minimum has an up/down or down/up arrangement of the two $\mathrm{H}$ atoms of the water moieties. From this minimum, a transition state is reached, in which one of the two hydrogen atoms is in plane. By further rotation of this $\mathrm{H}$ atom in the same direction, a second (local) minimum is reached, in which both $\mathrm{H}$ atoms point down(up)wards. Both local minima with the hydrogens pointing both up or both down have the same energy as the up/down and down/up conformations of the global minimum. The stationary points along this coordinate have been calculated at the RICC2/TZVP level of theory for both electronic states. All energies, relative to the global minimum, are given at the 


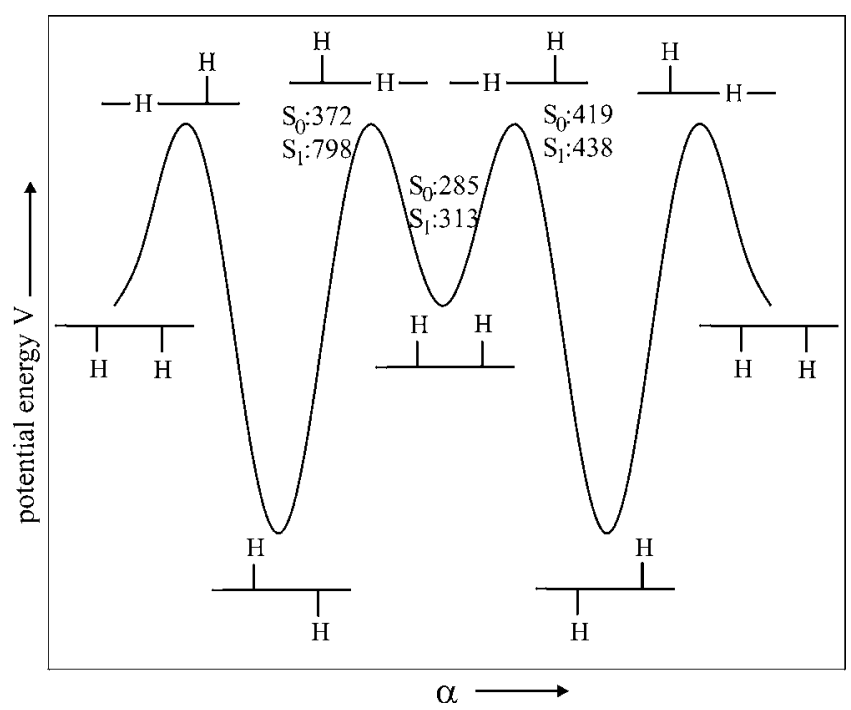

FIG. 5. One-dimensional potential energy curve of the tunneling coordinate in the 7-azaindole $\left(\mathrm{H}_{2} \mathrm{O}\right)_{2}$ cluster. The conformations of the stationary points have been depicted symbolically with the line of sight into the plane of the hydrogen bond ring and the azaindole monomer. The two out-of-plane hydrogen atoms are labeled by $8 \mathrm{~b}$ and $9 \mathrm{~b}$ in Fig. 3 .

respective stationary points in Fig. 5. From the relative energies of the transition states in Fig. 5, it is clear that the arrangement with the in-plane hydrogen atom at the pyrrolo side has a slightly different energy than at the pyridino side.

A normal mode analysis at the level of structure optimization has been performed to check the right number of imaginary and nonimaginary frequencies for the minima and the transition states. The splitting in the spectrum would then arise from two transitions, connecting the global and the local minima in each electronic state [cf. Fig. 6(a)]. Since the ground state energy difference between the two conformers amounts only to $285 \mathrm{~cm}^{-1}$ (including zero-point-energy correction) and the barrier separating them is calculated to be $372 \mathrm{~cm}^{-1}$, both conformers might be frozen out in the course

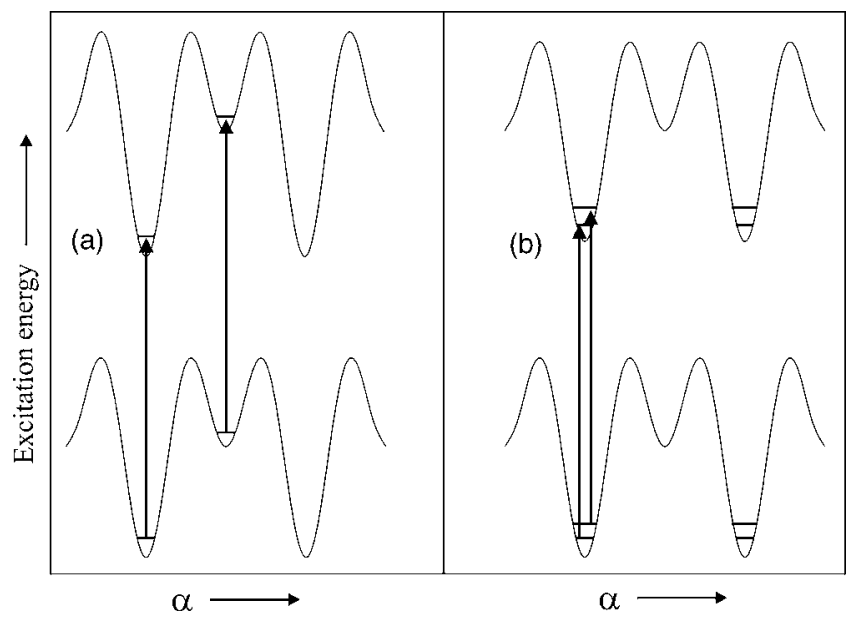

FIG. 6. Energy level scheme for the possible transitions in 7-azaindole $\left(\mathrm{H}_{2} \mathrm{O}\right)_{2}$. (a) Transitions between different conformers. The assumption is made that under the expansion conditions both conformers are frozen out simultaneously. (b) Transitions between different tunneling components of the most stable conformer, which in this model is frozen out exclusively in the supersonic expansion. of the expansion, forming an intensity ratio between the two bands which is governed by the expansion conditions.

If on the other hand, only the most stable conformer is exclusively frozen out in the supersonic expansion; an alternative explanation for the splitting can be given on the basis of a tunneling splitting of the lowest vibrational level in the potential energy curve of Fig. 5, which differs from the excited state splitting by the absolute value of the observed splitting.

In the following we estimate the order of magnitude of the splitting. Since the energy differences of the various transition states (at least in the electronic ground state) are small, it is possible to approximate the potential by an expansion in cosine terms,

$$
V_{4}=\frac{1}{2}\left(V_{2}(1-\cos 2 \alpha)+V_{4}(1-\cos 4 \alpha)\right),
$$

with $V_{2}=-290 \mathrm{~cm}^{-1}$ and $V_{4}=+200 \mathrm{~cm}^{-1}$ in the electronic ground state.

Using the potential given above and a reduced torsional constant $B$ of $23 \mathrm{~cm}^{-1}$, which is typical for a hydroxy torsion, ${ }^{23}$ a ground state torsional level splitting of $0.4 \mathrm{~cm}^{-1}$ is obtained using the analytical solutions from Ref. 24 of the Schrödinger equation with the Hamiltonian

$$
H_{T}=B \frac{d^{2}}{d \alpha^{2}}+\frac{1}{2} \sum_{n} V_{n}(1-\cos n \alpha),
$$

as implemented in the program HTORFIT, which is described in Ref. 25. For the excited state, a negligible splitting results due to the much higher barrier. Therefore, the very crude approximation that the potential in the excited state is also approximated by a sum of cosine terms does not influence the result. Inspection of Fig. 5 shows that, while the minima with the hydrogen pointing "ud" and "du" are exactly equal, there is a large difference in the relative transition state energies for the hydrogen atom at the pyrrolo side or at the pyridino side being in plane, respectively. Thus, a potential containing cosine and sine terms would be more appropriate. According to the fact that the barrier is much higher in the excited state a very small splitting would result in any of the model potential chosen. Regarding the fact that the above estimation contains a few very rough approximations, the appraised splitting is surprisingly close to the experimentally determined one. Nevertheless, one has to keep in mind that the estimation presented here is only meant to show the right order of magnitude for the splitting expected from the tunneling motion.

The two possible explanations for the splitting of the electronic origin of the 7-azaindole $\left(\mathrm{H}_{2} \mathrm{O}\right)_{2}$ cluster are depicted in Fig. 6. A distinction between these two possibilities seems to be difficult. In case (b), the relative intensities of the subbands will only depend on the spin statistical weights of the two components but not on the expansion conditions, since both transitions originate from the same ground state potential energy minimum. In case (a) the expansion conditions should alter the relative intensities. In none of the experiments we performed does a change of the relative intensities could be observed, which would point to case (b). Nevertheless, the range in which the expansion conditions 
can be varied without loosing the cluster signal are quite small for the 7-azaindole $\left(\mathrm{H}_{2} \mathrm{O}\right)_{2}$ cluster. Thus, at the present moment, no definite answer can be given to this question. Since the chance of the difference of the two excitations originating from different minima on the potential energy surface shown in Fig. 6(a) being as small as observed experimentally, we strongly prefer the tunneling model [case (b)].

\section{CONCLUSIONS}

The structures of the 7-azaindole $\left(\mathrm{H}_{2} \mathrm{O}\right)_{1,2}$ clusters were determined in the $S_{0}$ and the $S_{1}$ state. Both in the ground and excited state cyclic hydrogen bonded clusters of the $1 \mathrm{H}$ tautomer were found, although in the excited state the $7 \mathrm{H}$ tautomer is more stable one. Of course, this is not in the Franck-Condon region for transitions from the $1 H$ ground state.

Contrary to the case of the 7-azaindole monomer, we find the $S_{1}$ state in the microsolvated clusters to be of $L_{a}$ character in Platts nomenclature $(\mathrm{HOMO} \rightarrow \mathrm{LUMO})$. This conclusion can be drawn on the basis of the excited state structures, the transition dipole moment orientations and the adiabatic excitation energies.

The increased acidity of the pyrrolic NH group and the increased basicity of the pyridino $\mathrm{N}$ atom in the electronically excited state leads to a considerable shortening of both hydrogen bonds in the cyclic 7-azaindole $\left(\mathrm{H}_{2} \mathrm{O}\right)_{1}$ cluster.

Excited state lifetimes of $10 \mathrm{~ns}$ have been determined from the Lorentz contribution to the line profiles for both clusters, which are orders of magnitude larger that the two time constants for a biexponential decay, that has been measured by pump-probe spectroscopy.

The spectral splitting observed in the 7-azaindole $\left(\mathrm{H}_{2} \mathrm{O}\right)_{2}$ cluster can be explained on the basis of a tunneling motion, which interconverts the two out-of-plane hydrogen atoms of the hydrogen bond.

\section{ACKNOWLEDGMENTS}

This work has been performed in the SFB 663 TP A2, Universität Düsseldorf and was printed upon its demand with financial support from the Deutsche Forschungsgemeinschaft. We thank David Pratt and Karl Kleinermanns for helpful discussions. Granted computing time at the Austrian Grid network and computational facilities of the Johannes Kepler Universitat Linz are gratefully acknowledged.

${ }^{1}$ Y. N. Svartsov and M. Schmitt, J. Chem. Phys. 128, 214310 (2008).

${ }^{2}$ K. Fuke, H. Yoshiuchi, K. Kaya, Y. Achiba, K. Sato, and K. Kimura, J. Phys. Chem. 88, 5840 (1984).

${ }^{3}$ S. K. Kim and E. R. Bernstein, J. Phys. Chem. 94, 3531 (1990).

${ }^{4}$ A. Nakajima, M. Hirano, R. Hasumi, K. Kaya, H. Watanabe, C. C. Carter, J. Williamson, and T. A. Miller, J. Phys. Chem. A 101, 392 (1999).

${ }^{5}$ D. E. Folmer, E. S. Wisniewski, J. R. Stairs, and J. A. W. Castleman, J. Phys. Chem. A 104, 10545 (2000).

${ }^{6}$ H. Yokoyama, H. Watanbe, T. Omi, S. Ishiuchi, and M. Fujii, J. Phys. Chem. A 105, 9366 (2001)

${ }^{7}$ R. Brause, D. Krügler, M. Schmitt, K. Kleinermanns, A. Nakajima, and T. A. Miller, J. Chem. Phys. 123, 224311 (2005).

${ }^{8}$ A. Hara, K. Sakota, M. Nakagaki, and H. Sekiya, Chem. Phys. Lett. 407, 30 (2005)

${ }^{9}$ M. Schmitt, C. Ratzer, K. Kleinermanns, and W. L. Meerts, Mol. Phys. 102, 1605 (2004)

${ }^{10}$ C. Kang, J. T. Yi, and D. W. Pratt, J. Chem. Phys. 123, 094306 (2005).

${ }^{11}$ R. Brause, M. Schmitt, D. Spangenberg, and K. Kleinermanns, Mol. Phys. 102, 1615 (2004).

${ }^{12}$ C. Kang, J. T. Yi, and D. W. Pratt, Chem. Phys. Lett. 423, 7 (2006).

${ }^{13}$ M. Schmitt, J. Küpper, D. Spangenberg, and A. Westphal, Chem. Phys. 254, 349 (2000).

${ }^{14}$ S. Gerstenkorn and P. Luc, Atlas du Spectre d'Absorption de la Molécule d' iode $14800-20000 \mathrm{~cm}^{-1}$ (CNRS, Paris, 1986).

${ }^{15}$ W. L. Meerts, M. Schmitt, and G. Groenenboom, Can. J. Chem. 82, 804 (2004).

${ }^{16}$ W. L. Meerts and M. Schmitt, Phys. Scr. 73, C47 (2005).

${ }^{17}$ W. L. Meerts and M. Schmitt, Int. Rev. Phys. Chem. 25, 353 (2006).

${ }^{18}$ C. Ratzer, J. Küpper, D. Spangenberg, and M. Schmitt, Chem. Phys. 283, 153 (2002).

${ }^{19}$ M. Schmitt, D. Krügler, M. Böhm, C. Ratzer, V. Bednarska, I. Kalkman, and W. L. Meerts, Phys. Chem. Chem. Phys. 8, 228 (2006).

${ }^{20}$ J. Catalán and J. L. G. de Paz, J. Chem. Phys. 122, 244320 (2005).

${ }^{21}$ J. Catalán, P. Pérez, J. C. del Valle, J. L. G. de Paz, and M. Kasha, Proc. Natl. Acad. Sci. U.S.A. 99, 5799 (2002).

${ }^{22}$ Y. Huang, S. Arnold, and M. Sulkes, J. Phys. Chem. 100, 4734 (1996).

${ }^{23}$ G. Berden, W. L. Meerts, M. Schmitt, and K. Kleinermanns, J. Chem. Phys. 104, 972 (1996)

${ }^{24}$ J. D. Lewis, T. B. Malloy, Jr., T. H. Chao, and J. Laane, J. Mol. Struct. 12, 427 (1972).

${ }^{25}$ C. Jacoby and M. Schmitt, ChemPhysChem 5, 1686 (2004). 